\title{
PRÁTICAS DE MONITORIA ACADÊMICA NO CONTEXTO BRASILEIRO
}

\author{
ACADEMIC MONITORING PRACTICES IN THE BRAZILIAN \\ CONTEXT
}

\section{PRÁCTICAS DE MONITOREO ACADÉMICO EN EL CONTEXTO BRASILEÑO}

\author{
Juliane de Oliveira ${ }^{1}$ \\ http://orcid.org/0000-0001-7996-4133 \\ Dilmeire Sant'Anna Ramos Vosgerau ${ }^{2}$ \\ http://orcid.org/0000-0002-9508-0888
}

1 Pontifícia Universidade Católica do Paraná, Curitiba, Paraná - Brasil. E-mail:
juh_oliver87@hotmail.com.
2 Pontifícia Universidade Católica do Paraná, Curitiba, Paraná - Brasil. E-mail: dilmeire.vosgerau@pucpr.br.

\section{Resumo}

Esta revisão integrativa teve como objetivo identificar as propostas e os resultados de práticas de monitorias, tendências e seus desafios nas instituições de ensino superior. O levantamento foi realizado no ano de 2019, nas bases de dados SciELO e Educa, utilizando-se o descritor "monitoria" em qualquer parte do documento, totalizando 82 artigos. Restaram 12 documentos após a aplicação dos seguintes critérios de exclusão: a monitoria relatada não acontece em instituição de ensino; não é uma relação entre estudante ensinando outro estudante (monitoria acadêmica). Os documentos foram categorizados em: programas de monitoria institucionais, projetos de monitoria em disciplinas ou áreas, programa de formação de monitores. Os resultados evidenciam a escassez de pesquisas relacionadas com o tema; em contrapartida, os trabalhos existentes reforçam a importância da monitoria acadêmica como ferramenta estratégica para a melhora da qualidade de ensino. A monitoria não é uma estratégia de ensino fácil, trata-se de uma prática que exige acompanhamento e dedicação constantes tanto dos professores orientadores quanto dos monitores, porém a implementação de um programa de monitoria em uma instituição de ensino superior pode trazer muitos avanços para a formação e aprendizagem dos estudantes, futuros profissionais.

Palavras-chave: Monitoria Acadêmica. Graduação. Revisão Integrativa. 


\begin{abstract}
This integrative review aimed to identify the proposals and results of academic monitoring practices, trends and their challenges in higher education institutions. The survey was conducted in 2019, in the SciELO and Educa databases, using the descriptor "academic monitoring" in any part of the document, totaling 82 articles. There were 12 documents left after applying the exclusion criteria: the reported academic monitoring does not happen in an educational institution; it is not a relationship between student teaching another student (academic monitoring). The documents were categorized into: institutional monitoring programs, discipline or area monitoring projects, monitor training program. The results show the scarcity of research related to the theme; in contrast, the existing works reinforce the importance of academic monitoring as a strategic tool for improving the quality of teaching. Monitoring is not an easy teaching strategy, it is a practice that requires constant monitoring and dedication from both mentors and monitors. However, the implementation of an academic monitoring program in a higher education institution can bring many advances for the training and learning of students, future professionals.
\end{abstract}

Keywords: Academic Monitoring. University Graduate. Integrative Review.

\title{
Resumen
}

Esta revisión integradora tuvo como objetivo identificar las propuestas y los resultados de las prácticas de monitoreo, las tendencias y sus desafios en las instituciones de educación superior. La encuesta se realizó en 2019, en las bases de datos SciELO y Educa, utilizándose el descriptor "monitoreo" en cualquier parte del documento, totalizando 82 artículos. Quedaron 12 documentos después de aplicarse los criterios de exclusión: el monitoreo informado no ocurre en una institución educativa; no es una relación entre el alumno que enseña a otro alumno (monitoreo académico). Los documentos se clasificaron en: programas institucionales de monitoreo, proyectos de monitoreo de disciplina o áreas, programa de entrenamiento de monitoreo. Los resultados muestran la escasez de investigación relacionada con el tema; en contraste, los trabajos existentes refuerzan la importancia del monitoreo académico como una herramienta estratégica para mejorar la calidad de la enseñanza. El monitoreo no es una estrategia de enseñanza fácil, es una práctica que requiere monitoreo constante y dedicación tanto de los mentores como de los monitores. Sin embargo, la implementación de un programa de monitoreo en una institución de educación superior puede traer muchos avances para la capacitación y el aprendizaje de los estudiantes, futuros profesionales.

Palabras clave: Monitoreo Académico. Graduación. Revisión Integradora.

\section{Introdução}

A ideia do professor como único mediador do conhecimento é rompida pela prática de monitoria acadêmica. Essa prática de ensino é desenhada pela participação de estudantes e orientadores em diversos projetos, os quais são desenvolvidos para amparar o ensino de graduação (MEDEIROS, 2018). Nesses projetos, o estudante-monitor contribui para a 
aprendizagem de seu colega, assim como o aluno monitorado colabora para a aprendizagem do monitor, ou seja, ambos são participantes do processo ensino e aprendizagem.

A monitoria é entendida como um apoio ao processo pedagógico ao auxiliar na aprendizagem dos estudantes e, consequentemente, influenciar a melhora da qualidade do ensino (AMATO, 2016). Utilizada como estratégia de apoio ao ensino acadêmico, prevista na Lei de Diretrizes e Bases da Educação Nacional (LDBEN) n 9.394, de 20 de dezembro de 1996 (BRASIL, 1996), e em regimentos de algumas Instituições de Ensino Superior (IES), a monitoria permite uma articulação entre teoria e prática nos projetos em que é aplicada.

Além disso, coopera significativamente para a qualidade do ensino (MEDEIROS, 2018), propicia aos estudantes atingir os objetivos da disciplina e, aos monitores, a possibilidade de aprender a profissão docente (MOUTINHO, 2015), contribuindo também para reduzir dos índices de evasão e retenção dos cursos (AMATO, 2016).

A monitoria acadêmica não pode ser considerada apenas um local para a resolução de dúvidas, mas, sim, e sobretudo, um espaço de convívio e de trocas sociais (FLORES, 2018), constituindo-se em um ambiente livre de pressões, com tempo e horários suficientes para que a aprendizagem ocorra (FLORES, 2018), e favorável à ampliação do conhecimento proveniente da interação entre os estudantes, o que contribui para a sua formação (MOUTINHO, 2015).

A peça fundamental para um bom andamento da monitoria é o estudante-monitor, que é alguém, na maioria das vezes, mais experiente na tarefa, e responsável por auxiliar os colegas a pensarem sobre os conceitos, a pesquisarem de maneira autônoma e a perceberem as suas potencialidades e fragilidades (FLORES, 2018).

O monitor tem o papel de facilitador do aprendizado, isto é, ajuda os outros estudantes em suas dificuldades acadêmicas com uma linguagem mais próxima, porque também é um discente (MOUTINHO, 2015). Ademais, desempenha o papel de interlocutor, de mediador do que se aprende fora e dentro da sala de aula, colaborando com os seus pares (professores orientadores e demais estudantes da disciplina) e disseminando o conhecimento (MEDEIROS, 2018).

Prevista na Lei $\mathrm{n}^{\circ}$ 9.394, de 20 de dezembro de 1996, que compõe LDBEN, a figura do monitor se apresenta nos seguintes termos: "os discentes da educação superior poderão ser 
aproveitados em tarefas de ensino e pesquisa pelas respectivas instituições, exercendo funções de monitoria, de acordo com seu rendimento e seu plano de estudos" (BRASIL, 1996).

De maneira geral, a imagem do monitor representa os estudantes que, sob deliberadas condições, assumem a função de monitoria de uma determinada disciplina, atuando como extensão do corpo docente dentro da sala de aula (MANO, 2011), sendo que um monitor bem orientado pelo docente poderá ser capaz de construir conhecimentos e aprendizagens.

Considerando que o papel do monitor e as práticas de monitoria podem mudar conforme os regimentos das instituições de ensino e seus programas, a presente pesquisa tem por objetivo identificar as propostas e os resultados de práticas de monitoria nas IESs, buscando apresentar um cenário atual sobre as práticas de monitoria acadêmica, suas tendências e seus desafios, bem como colaborando para o desenvolvimento de novas pesquisas sobre o tema.

\section{Encaminhamento metodológico}

A fim de atender ao objetivo proposto, foi realizada uma revisão integrativa cujo "método proporciona a síntese de conhecimento e a incorporação da aplicabilidade de resultados de estudos significativos na prática" (SOUZA; SILVA; CARVALHO, 2010, p. 102). Ademais, “objetiva traçar uma análise sobre o conhecimento já construído em pesquisas anteriores sobre um determinado tema" (BOTELHO; CUNHA; MARCELO, 2011, p. 127). Para tanto foram utilizadas as seguintes fases de pesquisa (Quadro 1).

Quadro 1 - Fases da pesquisa

\begin{tabular}{|l|l|}
\hline \multicolumn{1}{|c|}{ Fases do processo } & \multicolumn{1}{c|}{ Aplicação } \\
\hline $\begin{array}{l}\text { Definição dos descritores para } \\
\text { direcionar as buscas a serem } \\
\text { realizadas }\end{array}$ & "Monitoria" (validada no Thesaurus Brasileiro da Educação [BRASED)] \\
\hline Definição das bases de dados & SciELO e Educa \\
\hline $\begin{array}{l}\text { Estabelecimento de critérios de } \\
\text { inclusão }\end{array}$ & Conter a palavra "monitoria" em qualquer parte do documento \\
\hline $\begin{array}{l}\text { Estabelecimento de critérios de } \\
\text { exclusão }\end{array}$ & $\begin{array}{l}1^{\circ} \text {.) a monitoria relatada não acontece em instituição de ensino; } \\
2^{\text {o }} \text {.) a monitoria relatada não é uma relação entre estudante ensinando outro } \\
\text { estudante }- \text { monitoria acadêmica }\end{array}$ \\
\hline Levantamento de dados & SciELO - 75 documentos encontrados \\
\hline
\end{tabular}




\begin{tabular}{|c|c|}
\hline & Educa -18 documentos encontrados \\
\hline $\begin{array}{l}\text { Exclusão de documentos } \\
\text { duplicados }\end{array}$ & 11 documentos excluídos \\
\hline $\begin{array}{l}\text { Aplicação do } 1^{\circ} \text { critério de } \\
\text { exclusão - leitura dos resumos }\end{array}$ & $1^{\circ}$ critério: 16 documentos selecionados de 82 (SciELO + Educa) \\
\hline Coleta do material de pesquisa & Download dos artigos completos selecionados \\
\hline $\begin{array}{l}\text { Aplicação do } 2^{\circ} \text { critério de } \\
\text { exclusão - leitura dos resumos }\end{array}$ & $2^{\circ}$ critério: 12 documentos selecionados \\
\hline $\begin{array}{l}\text { Leitura das publicações com } \\
\text { elaboração de síntese preliminar }\end{array}$ & $\begin{array}{l}\text { Leitura analítica dos artigos considerando-se as propostas, os objetivos, as } \\
\text { metodologias, os resultados e a área de pesquisa } \\
\text { Categorização } \\
\text { Elaboração de síntese (por meio de tabelas no Excel) }\end{array}$ \\
\hline $\begin{array}{l}\text { Organização do relatório do } \\
\text { estudo, compondo a } \\
\text { sistematização das sínteses e } \\
\text { identificando as tendências dos } \\
\text { temas abordados }\end{array}$ & $\begin{array}{l}\text { Organização das principais ideias e citações para a construção lógica do } \\
\text { artigo } \\
\text { Redação do texto }\end{array}$ \\
\hline $\begin{array}{l}\text { Análise e elaboração das } \\
\text { conclusões preliminares }\end{array}$ & $\begin{array}{l}\text { Apresentação das análises, resultados e conclusões da pesquisa } \\
\text { Finalização do texto }\end{array}$ \\
\hline
\end{tabular}

Fonte: Autoria própria, 2019.

A partir do objetivo de pesquisa foi definido o descritor "monitoria" para as buscas realizadas nas bases de dados SciELO e Educa em janeiro de 2019. Como critério de inclusão, essa palavra poderia ser encontrada em qualquer parte dos documentos localizados nas bases supracitadas, tendo sido alcançado um total de 93 artigos.

Após a identificação dos documentos que continham o descritor preestabelecido, foram excluídas as duplicidades (11 artigos), restando 82 documentos para a próxima etapa de análise.

Foi realizada, na sequência, a leitura dos resumos dos documentos restantes, com a intenção de selecionar apenas os artigos que abordam a monitoria nas instituições de ensino, o que resultou na escolha de 16 documentos.

Os 16 artigos foram lidos na íntegra a fim de se selecionarem apenas aqueles que cujo foco fosse a relação oficial de aprendizagem entre estudantes, definida nesta pesquisa como monitoria acadêmica, sendo selecionados 12 artigos (

\section{Quadro 2).}


Quadro 2 - Documentos selecionados

\begin{tabular}{|c|c|}
\hline Referências & Título \\
\hline $\begin{array}{l}\text { ALBUQUERQUE } \text { et al., } \\
2012 \mathrm{~b}\end{array}$ & $\begin{array}{l}\text { Bioquímica como sinônimo de ensino, pesquisa e extensão: um relato de } \\
\text { experiência }\end{array}$ \\
\hline $\begin{array}{l}\text { ALBUQUERQUE } \text { et al., } \\
\text { 2012a }\end{array}$ & $\begin{array}{l}\text { Monitoria de técnica operatória e cirurgia experimental e sua relevância na } \\
\text { formação médica }\end{array}$ \\
\hline ANDRADE et al., 2018 & $\begin{array}{l}\text { Contribuição da monitoria acadêmica para o processo ensino-aprendizagem } \\
\text { na graduação em Enfermagem }\end{array}$ \\
\hline BORSATTO et al., 2006 & $\begin{array}{l}\text { Processo de implantação e consolidação da monitoria acadêmica na UERJ e } \\
\text { na Faculdade de Enfermagem (1985-2000) }\end{array}$ \\
\hline DANTAS, 2014 & Monitoria: fonte de saberes à docência superior \\
\hline FRISON, 2016 & $\begin{array}{l}\text { Monitoria: uma modalidade de ensino que potencializa a aprendizagem } \\
\text { colaborativa e autorregulada }\end{array}$ \\
\hline $\begin{array}{l}\text { GARCIA; SILVA FILHO; } \\
\text { SILVA, } 2013\end{array}$ & $\begin{array}{l}\text { Monitoria e avaliação formativa em nível universitário: desafios e } \\
\text { conquistas }\end{array}$ \\
\hline HAAGI et al., 2008 & $\begin{array}{l}\text { Contribuições da monitoria no processo ensino-aprendizagem em } \\
\text { Enfermagem }\end{array}$ \\
\hline $\begin{array}{l}\text { NATÁRIO; SANTOS, } \\
2010\end{array}$ & Programa de monitores para o ensino superior \\
\hline PINHO et al., 2018 & $\begin{array}{l}\text { Monitoria e aprendizagem baseada em equipes: uma nova estratégia híbrida } \\
\text { para educação médica }\end{array}$ \\
\hline $\begin{array}{l}\text { SILVA; MARTINS } \\
\text { JUNIOR, } 2017\end{array}$ & $\begin{array}{l}\text { Desenvolvimento docente e monitoria de professores em formação com } \\
\text { apoio duma rede social: a experiência de licenciandos em Ciências com o } \\
\text { Facebook }\end{array}$ \\
\hline SOARES et al., 2003 & $\begin{array}{l}\text { Utilização de um serviço de monitoria virtual voltado para o ensino de } \\
\text { epidemiologia na graduação médica }\end{array}$ \\
\hline
\end{tabular}

Fonte: Autoria própria, 2019.

A partir da leitura e análise dos artigos selecionados, foi elaborada, com o apoio de uma planilha Excel $^{1}$, a codificação dos dados considerando as propostas, os objetivos, as metodologias, os resultados e as áreas de pesquisa.

Posteriormente, procedeu-se à análise de dados dos artigos selecionados por intermédio da técnica de categorização (BARDIN, 2010). Nessa fase da pesquisa, ponderando as propostas de monitoria nos estudos, foram definidas três categorias: programas de

\footnotetext{
${ }^{1}$ A planilha com a análise de dados poderá ser consultada acessando-se a base de dados do grupo de pesquisa CIDES (https://www.cidespesquisa.com.br).
} 
monitoria institucionais, projetos de monitoria em disciplinas ou áreas e programa de formação de monitores.

\section{Resultados}

Foi observado, por meio deste estudo, que as pesquisas publicadas sobre a prática de monitoria acadêmica na produção científica nacional ainda são escassas (12 artigos). As poucas existentes são, em sua maioria (oito artigos), pesquisas na área da saúde, seguida pela área da Educação (quatro artigos), conforme demonstrado no Quadro 33.

Quadro 3 - Áreas dos estudos

\begin{tabular}{|l|l|}
\hline \multicolumn{1}{|c|}{ Área } & \multicolumn{1}{|c|}{ Referências } \\
\hline Educação & $\begin{array}{l}\text { DANTAS, 2014; FRISON, 2016; GARCIA; SILVA FILHO; SILVA, 2013; SILVA; } \\
\text { MARTINS JÚNIOR, 2017 }\end{array}$ \\
\hline Saúde & $\begin{array}{l}\text { ALBUQUERQUE } \text { et al., 2012a; ALBUQUERQUE } \text { et al., 2012b; ANDRADE } \text { et al., 2018; } \\
\text { BORSATTO } \text { et al., 2006; HAAG } \text { et al., 2008; NATÁRIO; SANTOS, 2010; PINHO } \text { et al., } \\
2018 ; \text { SOARES } \text { et al., 2003 }\end{array}$ \\
\hline
\end{tabular}

Fonte: Autoria própria, 2019.

Ao se analisarem os objetivos de pesquisa dos 12 artigos selecionados, foi constatada a abordagem de outros temas além da monitoria acadêmica: quatro pesquisas (DANTAS, 2014; GARCIA; SILVA FILHO; SILVA, 2013; NATÁRIO; SANTOS, 2010; SILVA; MARTINS JÚNIOR, 2017) discorrem sobre a formação de professores e apresentam a monitoria como uma estratégia para desenvolver o potencial docente dos estudantes monitores; uma (FRISON, 2016) apresenta a aprendizagem colaborativa e analisa como a monitoria pode potencializá-la; uma (PINHO et al., 2018) trata de aprendizagem assistida por pares e aprendizagem baseada em equipe; duas (ALBUQUERQUE et al., 2012a; SOARES et al., 2003) tratam do ensino médico; uma (ALBUQUERQUE et al., 2012b) aborda ensino, pesquisa e extensão, relatando a experiência de monitores; duas (SILVA; MARTINS JUNIOR, 2017; SOARES et al., 2003) apresentam a monitoria sendo realizada em ambientes virtuais de aprendizagem (AVA), avaliando sua receptividade e verificando como se dá o aprendizado na metodologia da aprendizagem híbrida (blended learning) (SCHNEIDER et al., 2013), combinando os ensinos presencial e a distância. 
Entre os documentos analisados, quatro (ANDRADE et al., 2018; DANTAS, 2014; FRISON, 2016; HAAGI et al., 2008) consideram as percepções e as concepções de professores, estudantes e instituições acerca da prática de monitoria acadêmica e como ela pode potencializar o aprendizado e o desenvolvimento de habilidades nos estudantes; três (BORSATTO et al., 2006; GARCIA; SILVA FILHO; SILVA, 2013; PINHO et al., 2018) descrevem a implementação de projetos e do programa de monitoria; três relatam ou demonstram práticas de monitoria e suas contribuições (ALBUQUERQUE et al., 2012a, 2012b; NATÁRIO; SANTOS, 2010).

De todas as pesquisas analisadas, nove (ALBUQUERQUE et al., 2012a, 2012b; ANDRADE et al., 2018; DANTAS, 2014; FRISON, 2016; GARCIA; SILVA FILHO; SILVA, 2013; HAAGI et al., 2008; NATÁRIO; SANTOS, 2010; SILVA; MARTINS JUNIOR, 2017) abordam a importância da monitoria para o fortalecimento da relação entre teoria e prática para a formação profissional, cuja complexidade é firmemente discutida no meio acadêmico e em outros contextos educacionais nos quais ocorre o processo de ensino e aprendizagem (FERREIRA, 2014). Para Gamboa (2003, p. 125), "não é possível conceber a teoria separada da prática... É a própria relação entre elas que possibilita sua existência”.

Essa relação entre teoria e prática pode se dar por meio da monitoria em distintos espaços: salas de aula, bibliotecas, laboratórios, ambientes virtuais etc., contudo é fundamental que seja planejada para propiciar um ambiente com livre comunicação de ideias e de sentimentos, bem como confiança e cooperação mútua entre os envolvidos (NATÁRIO; SANTOS, 2010).

Quanto aos procedimentos metodológicos (Gráfico 1), prevalecem as pesquisas de abordagem qualitativa, pois, dos 12 artigos analisados, oito (ALBUQUERQUE et al., 2012b; ANDRADE et al., 2018; BORSATTO et al., 2006; DANTAS, 2014; FRISON, 2016; GARCIA; SILVA FILHO; SILVA, 2013; PINHO et al., 2018; SOARES et al., 2003) utilizam essa abordagem (corroborando, assim, a afirmação de André (2007) sobre os estudos qualitativos, que englobam um conjunto heterogêneo de métodos, técnicas e análises, os quais vem ganhando força no meio acadêmico); dois utilizam a abordagem quantitativa (ALBUQUERQUE et al., 2012a; HAAG et al., 2008); e dois são realizados a partir da abordagem quanti-qualitativa (NATÁRIO; SANTOS, 2010; SILVA; MARTINS JÚNIOR, 2017). 
Gráfico 1 - Procedimentos metodológicos

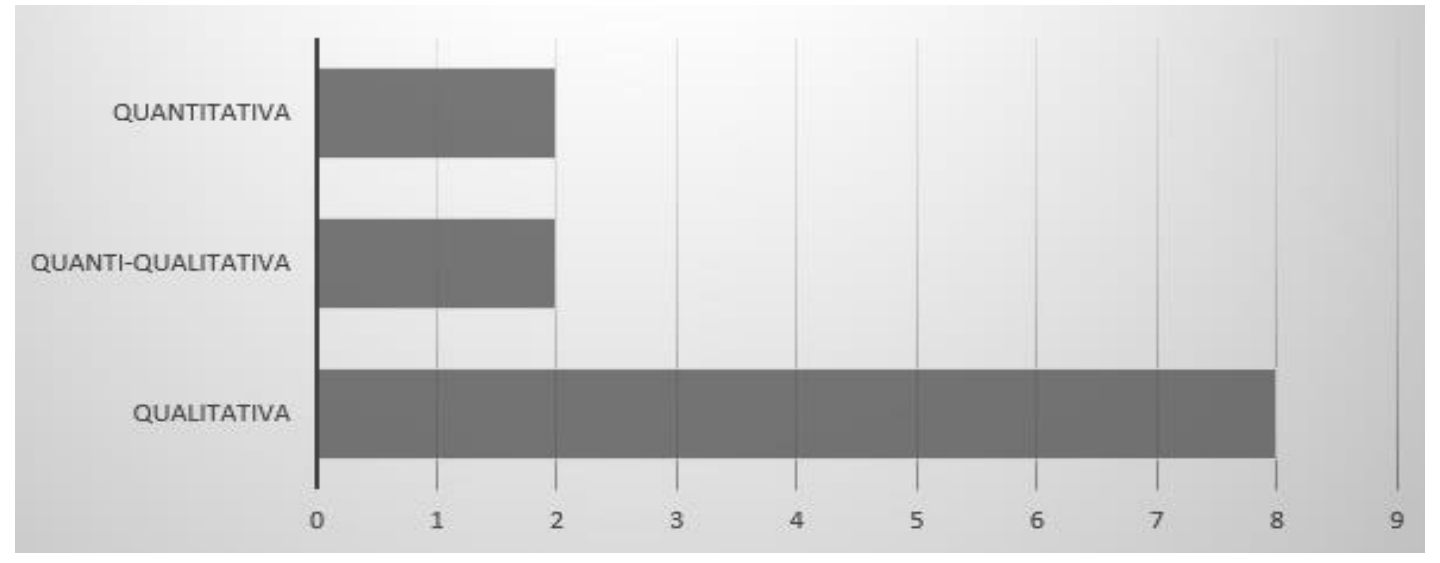

Fonte: Autoria própria, 2019.

Em relação aos enfoques metodológicos (Gráfico 1), seis artigos (ALBUQUERQUE et al., 2012a; ANDRADE et al., 2018; DANTAS, 2014; HAAG et al., 2008; NATÁRIO; SANTOS, 2010; PINHO et al., 2018) desenvolvem estudos descritivos; dois (ALBUQUERQUE et al., 2012b; SOARES et al., 2003) indicam terem realizado um relato de experiência; um (FRISON, 2016) configurou-se como exploratório; um (GARCIA; SILVA FILHO; SILVA, 2013) como pesquisa-ação; um (BORSATTO et al., 2006) como históricosocial; e um (SILVA; MARTINS JÚNIOR, 2017) como estudo de caso.

Gráfico 1 - Enfoque metodológico

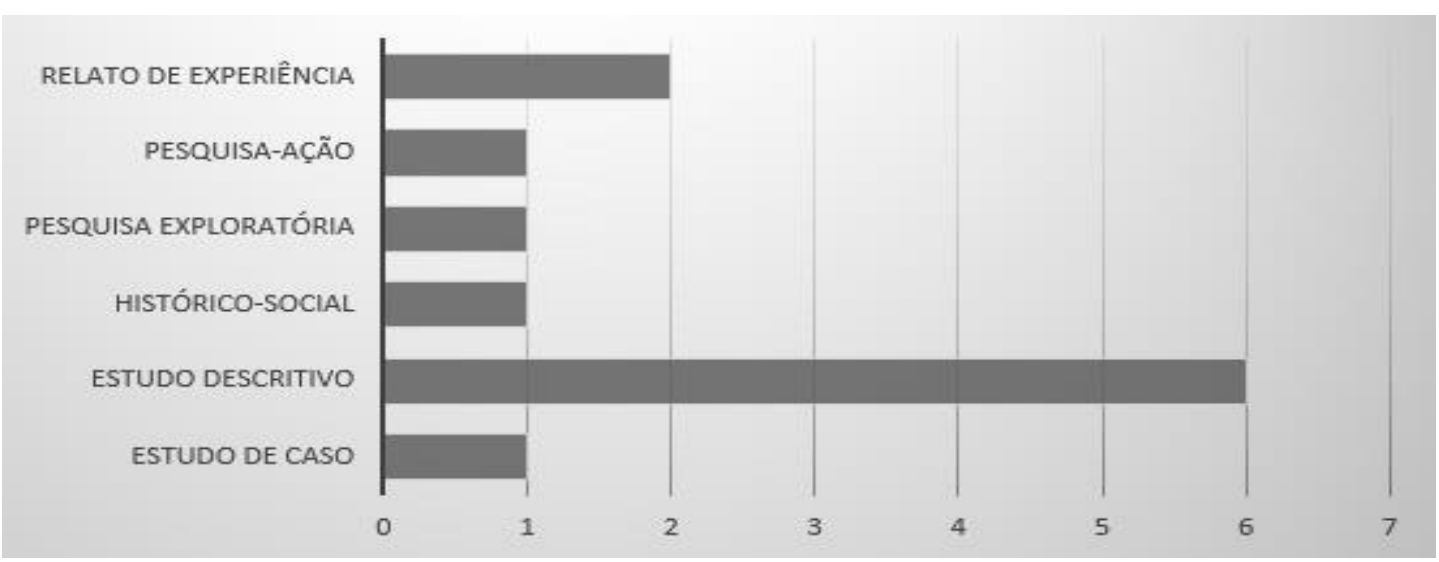


Fonte: Autoria própria, 2019.

Quanto aos participantes e às fontes de dados, prevalecem as vozes do estudantemonitor (oito pesquisas), do estudante monitorado (seis pesquisas) e do professor (cinco pesquisas), conforme demonstrado no Quadro 4.

Quadro 4 - Voz participante

\begin{tabular}{|l|l|}
\hline \multicolumn{1}{|c|}{ Voz participante } & \multicolumn{1}{|c|}{ Referências } \\
\hline Monitor & $\begin{array}{l}\text { ALBUQUERQUE } \text { et al., 2012a; ALBUQUERQUE } \text { et al., 2012b; DANTAS, 2014; } \\
\text { FRISON, 2016; GARCIA; SILVA FILHO; SILVA, 2013; HAAG } \text { et al., 2008; } \\
\text { NATÁRIO; SANTOS, 2010; PINHO } \text { et al., 2018 }\end{array}$ \\
\hline Estudante monitorado & $\begin{array}{l}\text { ANDRADE } \text { et } \text { al., 2018; FRISON, 2016; GARCIA; SILVA FILHO; SILVA, 2013; } \\
\text { HAAG } \text { et } \text { al., 2008; PINHO } \text { et al., 2018; SOARES } \text { et al., 2003 }\end{array}$ \\
\hline Professor & $\begin{array}{l}\text { ANDRADE } \text { et al., 2018; DANTAS, 2014; FRISON, 2016; GARCIA; SILVA } \\
\text { FILHO; SILVA, 2013; HAAG } \text { et al., 2008 }\end{array}$ \\
\hline
\end{tabular}

Fonte: Autoria própria, 2019.

A partir da análise de dados, foi possível categorizar as pesquisas selecionadas com base na apresentação das práticas de monitoria: programas de monitoria institucionais, projetos de monitoria em disciplinas ou áreas e programa de formação de monitores.

$\mathrm{Na}$ categoria "programas de monitoria institucionais", as pesquisas apresentam o programa de monitoria de suas instituições de ensino (BORSATTO et al., 2006; DANTAS, 2014; FRISON, 2016).

Borsatto et al. (2006) descrevem a trajetória de implantação do programa de monitoria da Universidade do Estado do Rio de Janeiro (UERJ) e da Faculdade de Enfermagem, analisam a consolidação dos seus processos no período de 1985-2000 e apresentam as resoluções institucionais do programa, descrevendo-as na prática como: requisitos para se tornar monitor, processo seletivo, carga horária da monitoria e atribuições do monitor. Os resultados evidenciam que o programa de monitoria da UERJ passou por diferentes momentos, os quais contribuíram para a sua consolidação. Foi possível verificar um processo crescente de normatização do programa, apresentando avanços em sua estrutura e dinâmica, como a criação do Departamento de Estágios e Bolsas/Cetreina, responsável por coordenar e controlar as atividades de monitoria acadêmica. Há evidências de avanço também no inter- 
relacionamento entre estudantes e professores e aprofundamento de conhecimentos nas disciplinas que participaram do programa.

Dantas (2014) busca delinear as concepções de duas universidades públicas federais (Universidade Federal do Rio Grande do Norte [UFRN] e Universidade de Brasília [UnB]) sobre o papel e a importância da monitoria para a docência superior de acordo com a lei brasileira e a visão de alguns professores e estudantes. Para isso apresenta as resoluções que normatizam os programas das IESs pesquisadas, descreve a prática de monitoria das duas instituições e compara alguns de seus processos. Os resultados indicam que o interesse pela monitoria proporciona incentivo à docência superior, experiência e possibilidade de o estudante-monitor enriquecer o seu currículo para além do interesse financeiro. A prática da monitoria leva os monitores a descobrirem suas próprias habilidades docentes na direção de uma formação responsável, articulando teoria e prática de modo consistente.

Frison (2016) analisa se a monitoria, entendida como uma modalidade de ensino, potencializa a aprendizagem colaborativa e autorregulada dos estudantes universitários. Para tanto descreve a prática de monitoria de uma IES particular. Os resultados revelaram que o trabalho realizado foi produtivo para a aprendizagem autorregulada de monitores e estudantes monitorados, pois a maior parte desses universitários aprendeu a utilizar diferentes estratégias de aprendizagem. Ademais, a monitoria tende ao sucesso nos espaços universitários porque investe em aprendizagem ativa, mediada, interativa e autorregulada.

Os programas institucionais de monitoria, apresentados nas três pesquisas anteriores, evidenciam resultados positivos em relação às práticas de monitoria, como: melhor aprendizado nas disciplinas que participam dos programas (BORSATTO et al., 2006; DANTAS, 2014; FRISON, 2016), melhores resultados nas avaliações (DANTAS, 2014; FRISON, 2016), diminuição do índice de reprovações (DANTAS, 2014), articulação entre teoria e prática (DANTAS, 2014; FRISON, 2016), melhor relacionamento entre professores e estudantes (BORSATTO et al., 2006; FRISON, 2016), colaboração entre os discentes (FRISON, 2016) e incentivo à docência superior (DANTAS, 2014). Nos dois primeiros estudos, no entanto, (BORSATTO et al., 2006; DANTAS, 2014), embora os programas de monitoria dessas instituições sejam bem delineados, o papel do estudante-monitor parece ser incompreendido tanto pelos monitores quanto pelos professores orientadores, que acabam posicionando esse discente como um auxiliar, gerando certa insatisfação. 
$\mathrm{Na}$ categoria "projetos de monitoria em disciplinas ou áreas", os estudos abordam projetos específicos de monitoria voltados a uma única disciplina ou disciplinas de uma determinada área (ALBUQUERQUE et al., 2012a; ALBUQUERQUE et al., 2012b; GARCIA; SILVA FILHO; SILVA, 2013; HAAG et al., 2008; PINHO et al., 2018; SILVA; MARTINS JÚNIOR, 2017; SOARES et al., 2003).

Albuquerque et al. (2012a) demonstram a evolução da monitoria de Técnica Operatória e Cirurgia Experimental (TOCE) da Faculdade de Medicina da Bahia (Universidade Federal da Bahia [UFBA] por intermédio da visão dos monitores que passaram pela estrutura antiga e pela estrutura nova em curso desde 2009 e apresentam o projeto de monitoria da disciplina e suas práticas, comparando os processos antigos com os atuais. Os resultados evidenciam que a maioria dos estudantes obteve o alcance total ou parcial de seus objetivos iniciais com a participação no projeto, considerando que as experiências e conhecimentos adquiridos foram e serão úteis para a sua prática médica. Além disso, concluem que o projeto de monitoria de TOCE tem evoluído positivamente na visão dos estudantes participantes.

Albuquerque et al. (2012b) apresentam o relato de experiência de quatro estudantes do curso de Medicina da Universidade Federal do Vale do São Francisco (UNIVASF) como monitores da disciplina de Bioquímica Metabólica. A proposta da prática de monitoria exposta nesse estudo é sustentada nos três pilares da universidade - ensino, pesquisa e extensão - e procura demonstrar a importância da formação acadêmica em uma visão indissociável dessas vertentes. Os resultados mostram que a proposta de monitoria para o ensino conseguiu desenvolver maior empatia dos docentes pela matéria, assim como o reconhecimento de melhor aproveitamento. A extensão possibilitou aos discentes um exercício para o desenvolvimento da habilidade de trabalhar no relacionamento com o cidadão. Com a proposta da pesquisa, foram possíveis a vivência de aulas práticas e o envolvimento com experiências, revelando a importância da conexão entre o ensino teórico e o ensino prático.

Garcia, Silva Filho e Silva (2013) analisam uma experiência de ensino desenvolvida na UFRN pela equipe de monitoria da disciplina Organização da Educação Brasileira. A equipe implementou uma proposta de avaliação formativa na turma com a intenção de envolver os estudantes no processo de ensino e aprendizagem, assim como discutir a importância do trabalho docente (todo o processo é relatado pelos autores). Os resultados 
revelam que a experiência da avaliação formativa colaborou para conscientizar os estudantes sobre a corresponsabilidade de todos os envolvidos no processo de ensino e aprendizagem e demonstram também a importância de se discutir o trabalho docente em seus cursos de formação.

Haag et al. (2008) investigaram a percepção do estudante e do professor em relação à prática de monitoria e à sua influência no desenvolvimento das atividades de estágio. Para isso descrevem a proposta prática dos projetos de monitoria das disciplinas Fundamentos de Enfermagem I e Socorros de Urgência desenvolvidas na Universidade do Vale do Rio dos Sinos (UNISINOS). Os estudantes relataram experiências positivas, das quais destacam-se: esclarecimento de dúvidas; atenção e acolhimento/didática dos monitores; liberdade para questionar e realizar atividades práticas; e autoconfiança e segurança proporcionadas pela combinação de conhecimentos teóricos com a habilidade prática. $\mathrm{O}$ estudo revela a prática da monitoria como uma ferramenta que facilita o desenvolvimento teórico-prático do aluno, mas não se concretiza como instrumento único e responsável pela sua qualificação.

Pinho et al. (2018) descrevem o teste piloto de um novo modelo de monitoria desenvolvido, então denominado método PAL-TBL (Peer-Assisted and Team-Based Learning - Aprendizagem Assistida por Pares e Aprendizagem Baseada em Equipe), aplicado durante as aulas de Fisiologia Humana com os estudantes do primeiro ano do curso de Medicina da Universidade de Pernambuco (UFPE) (toda a prática do projeto é relatada pelos autores). Os resultados mostram que a proposta levou os estudantes a refletirem mais sobre os problemas apresentados durante as discussões em sala de aula e estimulou o conhecimento prévio desses discentes, bem como sua capacidade de trabalho em grupo, permitindo uma rica experiência de aprendizagem.

Silva e Martins Júnior (2017) apresentam uma possibilidade para o acompanhamento monitorado de licenciandos em Ciências em uma rede social, o Facebook, usada como ambiente virtual formativo de aprendizagem. Os autores investigam o desenvolvimento docente desses estudantes com relação a cinco atividades semipresenciais realizadas, as quais foram descritas enquanto as respostas dos discentes foram discutidas entre os pesquisadores. Os resultados evidenciam a dialogicidade virtual obtida pela linguagem telecolaborativa e a práxis do professor como elemento contribuinte, desde que dirigida, por um planejamento adequado, para o ensino de Ciências. 
Soares et al. (2003) discutem o ensino de conteúdos de Saúde Coletiva do curso de Medicina da Faculdade de Medicina da Universidade Federal do Rio de Janeiro (UFRJ) com base em uma experiência de monitoria virtual. $\mathrm{O}$ trabalho apresenta a monitoria virtual como um instrumento complementar de ensino, descreve suas funcionalidades e mostra os resultados da avaliação da utilização e receptividade de seus serviços no período entre 2000 e 2002. Os resultados evidenciam que, dos $86 \%$ dos estudantes que avaliaram a monitoria virtual, $61 \%$ ficaram totalmente satisfeitos com as respostas recebidas, $18 \%$ afirmaram que a monitoria virtual aumentou sua frequência de uso da internet e $88 \%$ consideraram uma boa ideia a inclusão do serviço em outras disciplinas.

Das pesquisas supracitadas, a maioria não evidencia preocupação em apresentar informações sobre o regulamento de monitoria no qual o projeto está inserido (ALBUQUERQUE et al., 2012a; ALBUQUERQUE et al., 2012b; HAAG et al., 2008; PINHO et al., 2018; SILVA; MARTINS JÚNIOR, 2017; SOARES et al., 2003). Algumas comunicam o processo seletivo do monitor e seus critérios de seleção; outras partem diretamente para a apresentação da prática do projeto (ALBUQUERQUE et al., 2012a; ALBUQUERQUE et al., 2012b; HAAG et al., 2008; PINHO et al., 2018; SILVA; MARTINS JÚNIOR, 2017; SOARES et al., 2003).

Em seus resultados, retomam alguns benefícios da prática de monitoria, como: relação de troca de experiências e conhecimentos entre os discentes (PINHO et. al., 2018), empatia pela disciplina e comprometimento com ela(ALBUQUERQUE et al., 2012b; GARCIA; SILVA FILHO; SILVA, 2013), melhor aproveitamento da disciplina (ALBUQUERQUE et al., 2012a; ALBUQUERQUE et al., 2012b; GARCIA; SILVA FILHO; SILVA, 2013; HAAG et al., 2008), aumento na interatividade entre professores e estudantes (GARCIA; SILVA FILHO; SILVA, 2013), além de voltarem a evidenciar a importância da conexão ensino teórico e ensino prático (ALBUQUERQUE et al., 2012a; ALBUQUERQUE et al., 2012b; GARCIA; SILVA FILHO; SILVA, 2013; HAAG et al., 2008; SILVA; MARTINS JÚNIOR, 2017). Ainda, nos estudos de monitoria virtual, há o aumento da utilização da internet (SILVA; MARTINS JÚNIOR, 2017; SOARES et al., 2003).

Por meio da presente pesquisa, observa-se que as atividades de monitoria nas universidades têm sido voltadas para a prática de ensino, considerando a afinidade com a disciplina pleiteada e um bom desempenho acadêmico como pré-requisitos aos futuros monitores e, em alguns casos, o interesse pela profissão docente. Das 12 pesquisas analisadas, 
apenas três (ALBUQUERQUE et al., 2012b; BORSATTO et al., 2006; DANTAS, 2014) abordam o envolvimento do monitor com pesquisa e extensão.

A categoria "programa de formação de monitores" conta com uma única pesquisa (NATÁRIO; SANTOS, 2010). Natário e Santos (2010) criaram um programa para formação de monitores tendo em vista a intenção de investigar as contribuições desse programa para monitores da área da saúde de uma universidade particular do estado de São Paulo. A formação ocorreu em 10 encontros semanais e os principais pontos abordados foram sobre o esclarecimento da função e do papel do estudante-monitor, assim como seu interesse pela carreira docente. Os resultados revelam que, após a participação no programa, os estudantesmonitores apresentaram maior esclarecimento sobre o seu papel e função em relação ao vínculo da monitoria com a docência e mais interesse pela carreira docente.

Entre as 12 pesquisas analisadas, a de Natário e Santos (2010) é a única a demonstrar preocupação com a formação dos monitores, principalmente no que diz respeito ao papel por ele desempenhado, sendo isso de muita importância, uma vez que a clareza do papel e da função do monitor é fundamental para que ele não se transforme em mão de obra qualificada para desempenhar atividades como digitador, auxiliar de laboratório ou secretário do professor (NATÁRIO; SANTOS, 2010).

Embora seja um estudo bastante relevante, pois analisa a percepção de discentes e docentes sobre a monitoria acadêmica como ferramenta de fortalecimento do ensino e da aprendizagem e como possibilidade de transformação intelectual e social e na formação profissional, o artigo de Andrade et al. (2018) é o único que não faz menção a características/práticas de programas ou projetos de monitoria, portanto não atendeu aos critérios de qualquer das três categorias citadas anteriormente. Os autores consideram que o processo de ensino e aprendizagem deve ser regido pelo diálogo, pois é ele que faz que professores, monitores e estudantes monitorados aprendam em conjunto, processo fortalecido e impulsionado pela monitoria acadêmica.

\section{Considerações finais}

Na presente pesquisa, buscou-se identificar as propostas e os resultados de práticas de monitorias nas IESs, sendo possível conceber as articulações preponderantes a respeito do 
tema e as movimentações acadêmicas sob as quais a monitoria é utilizada como estratégia no processo de ensino e aprendizagem.

Os resultados das pesquisas analisadas evidenciam que a prática de monitoria acadêmica é de suma importância como apoio à melhoria da qualidade do ensino, traz benefícios tanto para o estudante-monitor quanto para o monitorado e favorece a superação de dificuldades que limitam a aprendizagem, o comprometimento com o próprio aprendizado, a construção do conhecimento e a socialização, a relação entre docente e discentes (monitor e monitorado), bem como a colaboração com os pares (os estudantes aprendem juntos). Por se encontrar na condição de estudante, o monitor consegue compreender as pretensões e as dificuldades de seus colegas, o que possibilita vínculos significativos entre monitor e monitorado, facilitando o aprendizado.

Não há dúvidas acerca das contribuições da monitoria como facilitadora do processo de ensinar e aprende, porém existem alguns pontos sobre os quais há a necessidade de reflexão e discussão antes da implementação de um projeto ou programa, tal como o papel do monitor, pois ainda parece ser desconhecido pelos envolvidos na monitoria. Assim, seria interessante que os programas investissem na formação desses estudantes. Outro ponto é a falta de envolvimento do monitor com a pesquisa e extensão - eixos importantes para sua formação-, evidenciada por meio dos resultados de pesquisas.

A monitoria não é uma estratégia de ensino fácil, posto que exige acompanhamento e dedicação constantes tanto dos professores orientadores quanto dos monitores, contudo a implementação de um programa de monitoria em uma IES pode trazer muitos avanços para a formação e a aprendizagem de estudantes, futuros profissionais.

Enfim, considerando a carência de pesquisas, em esfera nacional, e a relevância do tema para o meio acadêmico, reitera-se, nesta pesquisa, a necessidade de mais estudos que se empenhem em descobrir como se dão o processo de ensino e aprendizagem e as mediações que ocorrem na monitoria acadêmica.

\section{Referências}

ALBUQUERQUE, G. de S. et al. Monitoria de técnica operatória e cirurgia experimental e sua relevância na formação médica. Rev. Bras. Educ. Med., v. 36, n. 4, p. 564-569, 2012 a.

ALBUQUERQUE, M. A. C. et al. Bioquímica como sinônimo de ensino, pesquisa e 
extensão: um relato de experiência. Revista Brasileira de Educação Médica, v. 36, n. 1, p. 137-142, 2012b.

AMATO, D. T. Programa de monitoria no ensino superior: o estudo de caso no CEFET/RJ. 2016. 104 f. Dissertação (Mestrado em Sistemas de Gestão) - Universidade Federal Fluminense, Niterói, 2016.

ANDRADE, E. G. R. de et al. Contribuição da monitoria acadêmica para o processo ensinoaprendizagem na graduação em enfermagem. Revista Brasileira de Enfermagem, v. 71, p. 1596-1603, 2018.

ANDRÉ, M. Questões sobre os Fins e sobre os Métodos de Pesquisa em Educação. Revista Eletrônica de Educação, v. 1, n. 1, p. 199-31, 2007.

BARDIN, L. Análise de conteúdo. Lisboa: Edições 70, 2010.

BORSATTO, A. Z. et al. Processo de implantação e consolidação da monitoria acadêmica na UERJ e na Faculdade de Enfermagem (1985-2000). Esc. Anna Nery, v. 10, n. 2, p. 187-194, 2006.

BOTELHO, L. L. R.; CUNHA, C. C. de A.; MARCELO, M. O método da revisão integrativa nos estudos organizacionais. Gestão e sociedade, v. 5, n. 11, p. 121-136, 2011.

BRASIL. Lei n 9394, de 20 de dezembro de 1996. Diretrizes e Bases da Educação Nacional. Brasil, 1996.

DANTAS, O. M. Monitoria: fonte de saberes à docência superior. Revista Brasileira de Estudos Pedagógicos, v. 95, n. 241, p. 567-589, 2014.

FERREIRA, J. de L. A complexa relação entre teoria e prática pedagógica na formação de professores. In: FERREIRA, J. de L. (org.). Formação de professores: teoria e prática pedagógica. Petrópolis: Vozes, 2014. p. 33-49.

FLORES, J. B. Monitoria de cálculo e processo de aprendizagem: perspectivas à luz da Sócio-interatividade e da teoria dos três mundos da matemática. 2018. 226 f. Tese (Doutorado em Ciências e Matemática) - Pontifícia Universidade Católica do Rio Grande do Sul, Porto Alegre, 2018.

FRISON, L. M. B. Monitoria: uma modalidade de ensino que potencializa a aprendizagem colaborativa e autorregulada. Pro-Posições, v. 27, n. 1, p. 133-153, 2016.

GAMBOA, S. S. A contribuição da pesquisa na formação docente. In: REALI, A. M. D. M. R.; MIZUKAMI, M. D. G. N. (ed.). Formação de professores: tendências atuais. São Carlos: Edufscar, 2003. p. 116-130.

GARCIA, L. T. dos S.; SILVA FILHO, L. G. da; SILVA, M. V. G. da. Monitoria e avaliação formativa em nível universitário: desafios e conquistas. Perspectiva, v. 31, n. 3, p. 973-1003, 2013.

HAAGI, G. S. et al. Contribuições da monitoria no processo ensino-aprendizagem em 
enfermagem. Revista Brasileira de Enfermagem, v. 61, n. 2, p. 215-220, 2008.

MANO, G. C. de M. Experiências do grupal: cartografia do estilo na prática de monitoria. 2011. 107 f. Dissertação (Mestrado em Psicologia Social e Institucional) - Universidade Federal do Rio Grande do Sul, Porto Alegre, 2011.

MEDEIROS, L. D. G. C. de. Saberes da monitoria: Uma análise a partir do curso de pedagogia da Universidade Federal da Paraiba. 2018. 119 f. Dissertação (Mestrado em Políticas Públicas, Gestão e Avaliação da Educação) - Universidade Federal da Paraíba, João Pessoa, 2018.

MOUTINHO, P. M. N. Monitoria: sua contribuição para o ensino-aprendizagem na graduação em enfermagem. 2015. 60 f. Dissertação (Mestrado em Ciências) - Universidade de São Paulo, Ribeirão Preto, 2015.

NATÁRIO, E. G.; SANTOS, A. A. A. dos. Programa de monitores para o ensino superior. Estudos de Psicologia (Campinas), v. 27, n. 3, p. 355-364, 2010.

PINHO, G. C. et al. Monitoria e Aprendizagem Baseada em Equipes: Uma nova estratégia híbrida para Educação Médica. Rev. Bras. Educ. Med., v. 42, n. 3, p. 162-170, 2018.

SCHNEIDER, E. I. et al. Sala de Aula Invertida em EAD: uma proposta de Blended Learning. Revista Intersaberes, v. 8, n. 16, p. 68-81, 2013.

SILVA, J. M.; MARTINS JUNIOR, F. R. F. Desenvolvimento docente e monitoria de professores em formação com apoio duma rede social: a experiência de licenciandos em Ciências com o Facebook. Educação, Formação \& Tecnologias, v. 10, n. 1, p. 59-73, 2017.

SOARES, A. L. A. G. et al. Utilização de um Serviço de Monitoria Virtual Voltado para o Ensino de Epidemiologia na Graduação Médica. Physis, v. 13, n. 1, p. 39-58, 2003.

SOUZA, M. T. de; SILVA, M. D. da; CARVALHO, R. de. Revisão integrativa: o que é e como fazer. Einstein, v. 8, n. 1, p. 102-108, 2010.

Enviado em: 13/10/2019

Revisado em: 15/02/2021

Aprovado em: 04/03/2021

Publicado em: 15/06/2021 